\title{
The Development of Immobilization Matrices with Adjustable Density for Use in the Immobilization of Stationary-Phase Operating Microorganisms within Continuous Bioreactors
}

\author{
Robert W. M. Pott
}

\begin{abstract}
Stationary phase organisms can be entrapped in immobilization matrices to prevent their being 'washed-out' of a bioreactor. A consequence of immobilization is that these beads, or immobilization supports, tend to settle out of solution, since they are more dense than the surrounding media. To counter this more mixing energy must be added to the reactor in the form of agitation in order to keep the beads in suspension, and to prevent mass transfer limitations. One solution to this issue is to include low density glass microspheres within the immobilization matrix in order to bring the overall density of the matrix closer to that of the media, and so reduce mixing energy requirements. This article outlines experiments demonstrating the use of glass microspheres in the production of calcium alginate hydrogel beads (a commonly used immobilization matrix) and shows the effect their addition has on the circulation of beads within a thermosiphon as a test photobioreactor which has no direct mixing energy added, but approximately $0.5 \mathrm{~mW}$ of indirect mixing energy added through the absorbance of light. The demonstration of this methodology highlights a potentially industrially applicable method for immobilization-matrix modification, leading to better bioreactor volume utilization and lower mixing energy expenses.
\end{abstract}

Index Terms-Bioprocess engineering, cell immobilization, immobilization matrix, reactor design, continuous operation, thermosiphon.

\section{INTRODUCTION}

Within the field of bioprocess engineering, there are several bioproducts produced during the stationary phase of an organism (eg. Hydrogen production by Rhodopseudomonas palustris [1], ethanol production by yeast [2], butanol production by Clostridium sp. [3]. This mode of production can be utilized to develop a continuous process, where cells act simply as 'biocatalysts', continuously being fed substrate and continuously producing product, without necessarily increasing biomass concentration. However, a limitation to this form of operation is that cells can be 'washed-out' of a bioreactor, if the cells are not multiplying (an issue inherent in stationary phase operation) and if there is no mechanism for keeping the biomass separate from the reaction fluid. A solution to this, which is commonly used industrially, is to immobilize these non-multiplying biocatalysts onto, or into, solid supports, such as calcium alginate hydrogel beads [4].

Manuscript received May 15, 2016; revised August 17, 2016. This work was supported in part by the National Research Foundation of South Africa.

R. W. M. Pott is with the Department of Process Engineering, University of Stellenbosch, South Africa (e-mail: rpott@sun.ac.za).
Immobilization of bacteria is frequently used in biotechnology, as immobilization matrices can provide thermal and mechanical stability, allow retention of biomass for continuous reactor operation and can allow for more concentrated cell loading to reach higher volumetric rates of reaction than that achieved in free-cell cultures [5].

A suitable matrix should exhibit some key characteristics: i) the material should be biocompatible and non-toxic to the cells at all stages of its assembly, ii) it should be mechanically and chemically stable under the conditions employed, and iii) it should disperse as completely as possible within the reactor to allow for optimal mass transfer, maximum reactor volumetric productivity. And in the case of photosynthetic organisms, where a final condition is also required: iv) the matrix should be transparent to allow penetration of incident light [6].

A class of materials often used in the immobilization of cells are hydrogels. Hydrogels can be defined as water dispersed colloid gels, and are often composed of polymers in which cross-linked network chains prevent dissolution in water [7]. These materials have been used extensively in various biomedical applications, such as cell encapsulation [8], or the controlled release of biomolecules [9]. These gels often exhibit a macroporous structure, a property which can allow quick diffusion of substrates and products into and out of the solid [10]. Furthermore, many of these colloids display several of the properties of an ideal immobilization candidate, listed above: they are often produced under physiological temperatures, and seldom require toxic chemicals in their production. Several types of hydrogel are transparent. Although some hydrogels can be degraded under certain $\mathrm{pH}$ or ionic conditions, many hydrogels exhibit excellent mechanical and chemical stability. Alginate, a seaweed derived set of carbohydrate polymers, is one the most widely used materials for immobilization and microencapsulation technologies ${ }^{11}$. Alginate supports are usually made by crosslinking the carboxyl groups with a cationic crosslinker such as $\mathrm{Ca}^{2+}, \mathrm{Ba}^{2+}$, or poly(l-lysine) [12], [13]. This produces semi-transparent hydrogels with good mechanical stability, although there are some limitations on their chemical stability in a number of reaction fluids.

While hydrogels are closer in density to the growth medium or reaction fluid than some other immobilization matrices, such as ceramics [14] or polyurethane foam [15], they do still tend to settle out of suspension without the addition of significant mixing energy. While some processes operate such that the immobilized cells are not required to circulate in the reactor, there are some systems which do: for example in a photobioreactor the immobilized cells all need 
to experience and absorb light, which necessitates continuously agitated immobilization beads.

There has been little work demonstrating the development of variable density hydrogels, especially in the context of cell immobilization or use in reactor and process development. One method which can be used to reduce the overall density of hydrogels is the addition of low density solids in suspension to the precursor mixture. Ideally such an additive should have a significantly lower density than water, be non-reactive, biologically compatible, sterilizable (if a sterile matrix is required), be chemically stable, and allow the penetration of light (if a photosynthetic organism is to be immobilized). Hollow glass microspheres (HGMs) fulfill these requirements.

HGMs are thin walled $(0.5-2.0 \mu \mathrm{m})$, roughly spherical silicate-glass particles, with diameters generally in the range of 10 to $200 \mu \mathrm{m}$, although larger spheres can be produced. The key characteristic for this application is that they generally have comparatively low real, and bulk, densities, which vary from 80 to 700 and 70 to $400 \mathrm{~kg} / \mathrm{m}^{3}$, respectively, as a function of the brand and selection of HGM [16]. The addition of these particles to the immobilization matrix will then decrease the overall density, such that gels of negative, neutral or positive buoyancy can be produced [17], depending on the process requirements.

One reactor system, commonly used in household solar water heating, is the thermosiphon. A thermosiphon utilizes the heat energy added to a fluid exposed to light to drive a passive circulation through the reactor volume [18]. Such systems utilize no direct mixing energy addition, and so can be used in this study to make the case for the low mixing energy requirements of appropriately composed HGM amended hydrogels.

This study will therefore focus on demonstrating how, through the addition of various amounts of HGMs to the hydrogel precursor solution, a population of hydrogel beads of variable density can be produced. This methodology will be demonstrated in a thermosiphon reactor, as a system which has very low mixing energy, in order to show that effective hydrogel distribution can be achieved even in reactor systems with very low mixing energy input, if hydrogels amended with HGMs are utilized.

\section{MATERIALS AND MethodS}

\section{A. Alginate Hydrogel Formation}

A stock solution of $5 \% \mathrm{wt} / \mathrm{v}$ sodium alginate in water was made up by dissolving the appropriate weight of sodium alginate powder (Sigma-Aldrich) in distilled water by heating the water to $\sim 80^{\circ} \mathrm{C}$ and continuously stirring for 30 minutes.

The alginate solution was separated into $100 \mathrm{ml}$ aliquots, to which was added varying masses of HGBs (Q-cell 6019 glass bubbles, $\rho=0.19 \mathrm{~g} / \mathrm{cm}^{3}$, size 5.0 to $175 \mu \mathrm{m}$, mean size $75 \mu \mathrm{m}$ ), between 0 to $0.08 \mathrm{~g} \mathrm{HGBs} / 1$ solution. These solutions were homogenized by vigorous mixing.

The system, as depicted in Fig. 1, was used to produce hydrogel beads from these precursor solutions. This was performed by using a peristaltic pump to drip the well mixed solution through a 12 gauge hypodermic needle from a height of $10 \mathrm{~cm}$ at a flow rate of approximately $5 \mathrm{ml} / \mathrm{min}$, into a $2 \%$ wt/v $\mathrm{CaCl}_{2}$ solution, being gentle agitated by magnetic stirrer.

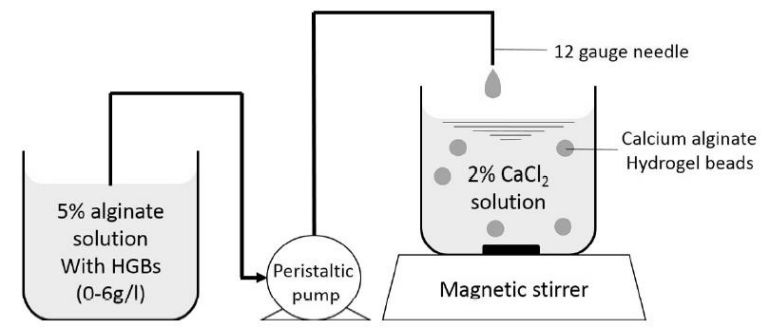

Fig. 1. schematic diagram depicting the production of calcium alginate hydrogel beads from sodium alginate solution.

The hydrogel beads were collected and left in the $2 \%$ $\mathrm{CaCl}_{2}$ solution overnight, to allow complete penetration of the $\mathrm{Ca}^{2+}$ ions into the solids. The beads were then drained from the solution, and left in a large excess of distilled water under agitation overnight, in order to remove any excess ions.

\section{B. Bead Size Selection}

In order to use beads within a small size distribution for further experimentation, a set of sizing sieves (Endecotts test sieves, sizes: $4.0 \mathrm{~mm}, 3.35 \mathrm{~mm}, 2.8 \mathrm{~mm}$, and $2.0 \mathrm{~mm}$ ) were utilized. Beads which did not fall within the required sizing $(3.35 \mathrm{~mm}<$ bead diameter $<4 \mathrm{~mm})$ were discarded.

\section{Density Determination}

The density of the beads, with their varying concentrations of HGBs, was determined by removing the excess external water from a sample of beads. The sample was then weighed using an analytical balance. The volume of the sample was then determined by displacement of water. The density was then calculated.

\section{Thermosiphon Reactor Set up and Operation}

The test reactor used to demonstrate minimal mixing energy requirements was a thermosiphon system. The thermosiphon consisted of a 2 liter working volume, made up of a $2.0 \mathrm{~cm} \mathrm{ID,} 105 \mathrm{~cm}$ long transparent riser tube, a $2.0 \mathrm{~cm}$ ID, $95 \mathrm{~cm}$ long shaded downcomer tube, and a shaded funnel collector. Within the collector volume a cooling coil was positioned, containing flowing cooling water at $20 \pm 1{ }^{\circ} \mathrm{C}$, pumped from a nearby cooling water cistern, while the water within the thermosiphon equilibrated to $25 \pm 1{ }^{\circ} \mathrm{C}$. The light to drive the system was provided by a $1500 \mathrm{~W}$ halogen light, positioned $20 \mathrm{~cm}$ from the riser tube.

\section{E. Thermosiphon Flow Characterization}

The fluid velocity in the downcomer and riser was tested by spiking the circulating fluid with a dye tracer. The time taken for the dye to circulate back to the collector was then measured.

Average mixing time in the whole system was also determine by filling the reactor with $0.01 \mathrm{M} \mathrm{HCl}$ dosed with phenolphthalein. Once the reactor was running at equilibrium a slug of $0.1 \mathrm{M} \mathrm{NaOH}$ was added, and the time taken for the indicator color to completely disappear from the reactor was measured.

\section{F. Bead Behavior within the Thermosiphon}

A mass of approximately $25 \mathrm{~g}$ beads was added to the thermosiphon, and the system allowed to equilibrate over 30 minutes. After equilibration the beads were separated into 
three categories: i) 'Sinkers' (S) - those beads which sink to the bottom of the reactor, and did not circulate, ii) 'Circulators' (C) - those beads which continually circulate within the reactor, and iii) 'Floaters' (F) - those beads which float on the surface and did not circulate. The number of each bead type (i, ii, and iii) was determined for each bead composition.

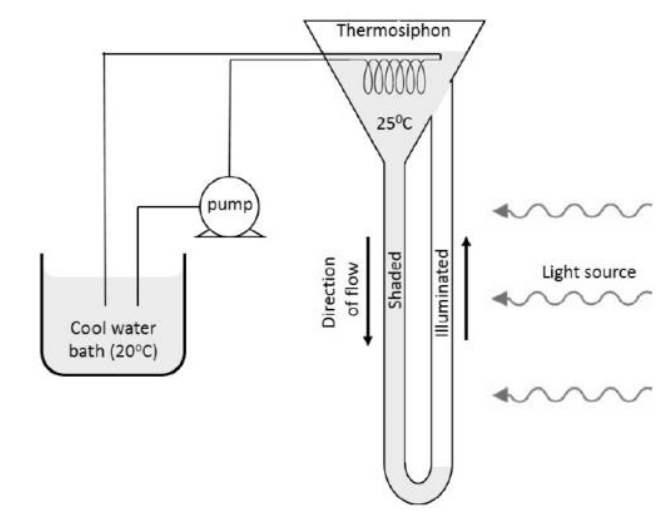

Fig. 2. Schematic representation of the test thermosiphon.

\section{RESULTS AND DISCUSSION}

The hydrogel beads formed by this methodology tend to be very uniform in size, and fairly close to spherical in shape. Of the bead samples produced (even with differing HGB compositions) $97 \%_{\text {mass }}$ fell within the sieving size $3.35 \mathrm{~mm}$ to $4 \mathrm{~mm}$, with the remainder both larger and smaller. The size of the beads is a function of the viscosity of the precursor solution (which itself is a function of temperature), the flow rate, and the exit tip diameter. In this study, all of these variables were fixed. Since the precursor solution spends a significant amount of time falling before encountering the gelation solution, the internal cohesion of the solution has sufficient time to form a roughly spherical ball. While the sphericity of the bead populations were not directly determined in this study, previous work has shown that this method produces relatively spherical beads.

Since the HGMs added to the precursor solution tend to float, even when dispersed in the viscous alginate solution, it is imperative that there is good mixing of the precursor solution to keep the solution as close to homogenous as practicable. There is, however, some variation in the HGM composition of the beads, even within a population formed from the same precursor solution, since some separation of HGMs and solution is inevitable. For this reason the beads that were produced had a slightly variable density, following a population curve within the sample centered on the bulk solution HGM concentration. For this reason there is limited utility in reporting the density of each population of beads, since such a measurement would aggregate the density distribution, and it is the density of each bead individually which determines its behavior in the test reactor, or under general agitation. Instead, a proxy measure is used - the behavior of the beads within the test reactor - as an indication of the density spread within each sample population.

The test reactor used in this study was a model thermosiphon reactor, which utilizes the absorbance of heat from a light source (generally sunlight, in non-lab scale set-ups, but from a halogen lamp in this case), in order to cause a density difference which drives passive circulation. In this reactor, since the circulation energy can be directly measured using the flow rate exiting the riser, the system is useful to determine mixing energy directly. This calculated mixing energy can then be used as an indicator for the energy required to cause the suspension and distribution within the reactor volume of the hydrogel beads.

The samples of hydrogels, containing varying concentrations of HGMs, were added to the thermosiphon reactor and the system allowed to equilibrate. For this reactor set-up, equilibrium occurred where the reactor temperature reached $25^{\circ} \mathrm{C}$; at this point all added heat was removed by the cooling coil flowing cooling water at a constant $20^{\circ} \mathrm{C}$. Under these conditions each sample of beads split itself into three distinct groups - Floaters, Circulators and Sinkers. For our purposes, we aim to maximize the number of Circulators, while minimizing both the Floaters and Sinkers, by adding the appropriate amount of HGMs to the precursor mixture. For each composition, the \% split between F, C and S was determined, and the results are shown in Fig. 3.

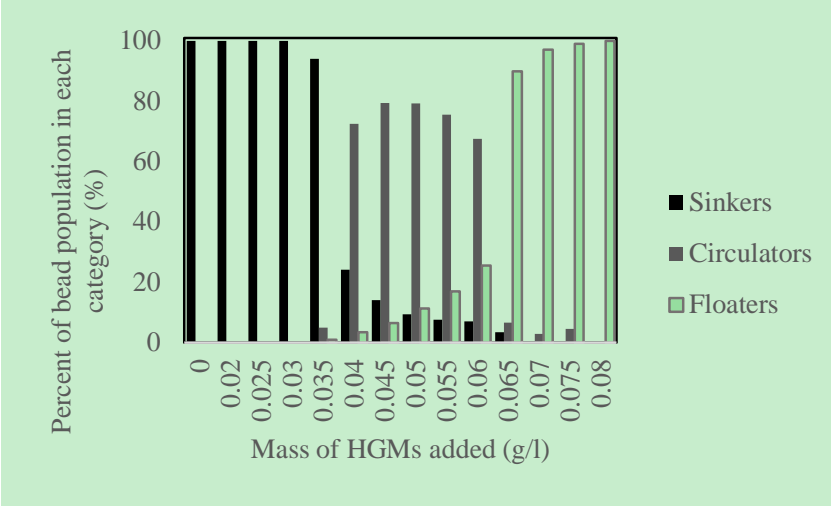

Fig. 3. The percentage split between Floaters, Circulators, and Sinkers for each hydrogel composition when operated in a test thermosiphon.

In the figure it is clear that a hydrogel composition including below $0.035 \mathrm{~g} / \mathrm{l} \mathrm{HGMs} \mathrm{no} \mathrm{circulation} \mathrm{takes} \mathrm{place,}$ since all the added beads simply sink. With an increase in mixing energy addition, forcing a flow through the reactor, these beads could be induced to circulated, with the unmodified alginate beads requiring the most energy.

Above the concentration of $0.065 \mathrm{~g} / \mathrm{l} \mathrm{HGMs}$, the proportion of Floaters quickly increases. The density of these beads will be greater than water, at this temperature, and since they float, only a very significant amount of added mixing energy would bring them within the remainder of the reactor volume. Thus, when adding HGMs to hydrogel solutions, it is important to determine the point at which the bulk density of the hydrogels drops below the reaction fluid, since beads composed of lower density hydrogels will no longer be able to circulate in the reactor volume, without significant mixing energy addition.

Between compositions of 0.04 and $0.06 \mathrm{~g} / \mathrm{l} \mathrm{HGMs}$, a significant percentage $(60-80 \%)$ of each bead population does continually circulate in the reactor. In these cases, the density of the beads is high enough that they settle down, and re-enter the downcomer, but low enough that the lift force caused by the thermosiphon effect is sufficient to overcome the gravitational settling force. Thus, when producing a hydrogel precursor solution, the aim should be to include sufficient MGBs such that the mixing energy added to the 
system is sufficient to circulate most of the added hydrogel beads. This minimum mixing energy, or threshold energy, can be determined using the apparatus as set up in this experiment, varying the driving force (temperature difference), to vary mixing energy addition. The mixing energy of this thermosiphon system manifests itself in the form of fluid velocity in the downcomer and riser.

The maximum velocity of the fluid flowing through the downcomer and riser was calculated as $0.2 \pm 0.06 \mathrm{~cm} / \mathrm{s}$, from the time taken for the fastest portion of the tracer slug to reach the exit. Using this velocity as an estimate for the maximum linear velocity for the system, the maximum Reynolds number for the system can be found to be $\mathrm{Re}<<2000$, which falls within the laminar flow regime. Since the flow is laminar, the flow within the downcomer and riser is characterized by the maximum velocity $\left(u_{\max }\right)$, such that the average linear velocity $\left(u_{\text {avg }}\right)$ is:

$$
u_{\text {avg }}=u_{\max } / 2
$$

Using the velocity profile we can calculate the kinetic energy added to the fluid originating in the heat energy from the lamp. This kinetic energy term can be used as an indirect mixing energy term, which can then provide some information on the mixing energy threshold for the circulating HGM-amended hydrogels.

$$
K E=\frac{1}{2} m\left(u^{2}\right) \text { avg }
$$

where $K E$ is kinetic energy, $m$ is mass and $u$ is the linear fluid velocity. The linear fluid velocity varies as a function of radius $(r)$, taking into account the maximum fluid velocity, at the center of the pipe, $\left(u_{\max }\right)$ and the pipe radius $(R)$.

$$
u(r)=u_{\max }\left[1-\frac{r^{2}}{R^{2}}\right]
$$

So, by integrating we get:

$$
\left(u^{2}\right)_{a v g}=\frac{u_{\max }^{2}}{3}
$$

And so

$$
K E=\frac{\pi r^{3} \rho_{\text {water }} u_{\text {max }}{ }^{2}}{12}
$$

Substituting in our calculated fluid velocity, and using $\rho_{\text {water }\left(25^{\circ} \mathrm{C}\right)}=0.997$, we find $0.5 \pm 0.1 \mathrm{~mW}$ of mixing energy being added to the system from the incident light. This energy, which is extremely small in comparison the mixing energy usually added to bioreactors by impellers of sparging, which tends to be 4 or 5 orders of magnitude larger, indicates the threshold for the minimum mixing energy required by the HGM amended hydrogels. In this case we can see from Fig. 3 that hydrogels composed of $5 \% \mathrm{wt} / \mathrm{v}$ alginate and 0 to $0.035 \mathrm{~g} / \mathrm{l}$ HGMs have a greater threshold mixing energy than $0.5 \mathrm{~mW}$, since circulation of the beads is not seen. While a composition above $0.035 \mathrm{~g} / \mathrm{l}$ HGMs has a threshold lower than $0.5 \mathrm{~mW}$, although this analysis is further complicated by the fact that a second effect, floatation, must be overcome by another mixing energy above a composition of $0.06 \mathrm{~g} / \mathrm{l}$ HGMs.

While the above derivation holds well for the fluid moving through the downcomer and riser, and so gives valuable information about the threshold energy required to circulate the various composition beads, this energy does not characterize the mixing in the total volume of the reactor. One measure which gives information about the fluid dynamics of the full reactor is the time taken for a slug of high $\mathrm{pH}$ solution to be dispersed sufficiently that is no longer deprotonates an indicator (phenolphthalein in this case). Performing this measurement gives a mixing time estimate of $575 \pm 63$ seconds, which is significantly longer than the 106 seconds for the fluid to pass through the downcomer and riser. This indicates that the mixing is comparatively slow in the collector, in comparison to the downcomer and riser. In that portion of the reactor the mixing energy is below the threshold for keeping the beads in suspension, which allows them to settle into the downcomer once more.

\section{CONCLUSION}

The results shown in this study indicate that hydrogels can indeed be fabricated, by the addition of HGMs, such that they have variable density, though a population distribution of densities was found within each composition, owing to the tendency of the HGMs to separate themselves from the bulk fluid. The application of the hydrogels within the thermosiphon demonstrated the use of these hydrogels in a system which provides very little mixing energy, illustrating that these modified hydrogel beads can be kept in suspension with the addition of a reduced amount of mixing energy.

\section{ACKNOWLEDGMENT}

With thanks to the NRF and the University of Stellenbosch for their financial contributions to the project.

\section{REFERENCES}

[1] R. W. M. Pott, C. J. Howe, and J. S. Dennis, "Photofermentation of crude glycerol from biodiesel using Rhodopseudomonas palustris: Comparison with organic acids and the identification of inhibitory compounds," Bioresour. Technol., vol. 130, pp. 725-730, 2013.

[2] M. Wada, J. Kato, and I. Chibata, "Continuous production of ethanol using immobilized growing yeast cells," Eur. J. Appl. Microbiol. Biotechnol., vol. 10, pp. 275-287, 1980.

[3] S. Y. Leeet al., "Fermentative butanol production by Clostridia," Biotechnol. Bioeng., vol. 101, pp. 209-228, 2008.

[4] J. Villadsen, J. Nielsen, and G. Lidén, Bioreaction Engineering Principles, Springer US, 2011.

[5] A. Coiffier, T. Coradin, C. Roux, O. M. M. Bouvet, and J. Livage, "Sol-gel encapsulation of bacteria: A comparison between alkoxide and aqueous routes," J. Mater. Chem., vol. 11, pp. 2039-2044, 2001.

[6] R. W. M. Pott, The bioconversion of waste glycerol into hydrogen by Rhodopseudomonas palustris, Ph.D thesis. Cambridge University, UK, 2014.

[7] T. Tanaka, Gels. Scientific American, pp. 124-138, 1981.

[8] N. Peppas, Hydrogels in Medicine and Pharmacy, CRC Press, 1987.

[9] W. E. Hennink and C. F. van Nostrum, "Novel crosslinking methods to design hydrogels,"Adv. Drug Deliv. Rev., vol. 64, pp. 223-236, 2012.

[10] M. C. Gutiérrez et al., "Hydrogel scaffolds with immobilized bacteria for 3D cultures," Chem. Mater., vol. 19, pp. 1968-1973, 2007.

[11] G. Fundueanu, C. Nastruzzi, A. Carpov, J. Desbrieres, and M. Rinaudo, "Physico-chemical characterization of Ca-alginate microparticles produced with different methods," Biomaterials, vol. 20, pp. 1427-1435, 1999.

[12] K. I. Draget, G. Skjåk-Bræk, and O. Smidsrød, "Alginate based new materials," Int. J. Biol. Macromol., vol. 21, pp. 47-55, 1997.

[13] O. Smidsrød and G. Skjåk-Bræk, "Alginate as immobilization matrix for cells," Trends Biotechnol., vol. 8, pp. 71-78, 1990 
[14] H. Nagadomi, T. Kitamura, M. Watanabe, and K. Sasaki, "Simultaneous removal of chemical oxygen demand (COD), phosphate, nitrate and $\mathrm{H} 2 \mathrm{~S}$ in the synthetic sewage wastewater using porous ceramic immobilized photosynthetic bacteria," Biotechnol. Lett., vol. 22, pp. 1369-1374, 2000.

[15] A. S. Fedorov, A. A. Tsygankov, K. K. Rao, and D. O. Hall, "Hydrogen photoproduction by Rhodobacter sphaeroides immobilised on polyurethane foam." Biotechnol. Lett., vol. 20, pp. 1007-1009.

[16] V. V. Budov, "Hollow glass microspheres, use, properties, and technology (Review)," Glas. Ceram., vol. 51, pp. 230-235, 1995.

[17] A. Laskoskie, D. Vesper, and H. Edenborn, "Hydrogel tracer beads: the development, modification, and testing of an innovative tracer for better understanding LNAPL transport in Karst Aquifers," in Proc. GSA Annual Meeting and Exposition, 2012.

[18] A. I. Kudish, P. Santamaura, and P. Beaufort, "Direct measurement and analysis of thermosiphon flow," Sol. Energy, vol. 35, pp. 167-173, 1985 .

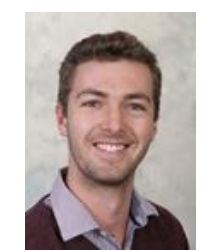

Robert W. M. Pott was born in Nelspruit, South Africa in 1986. He completed a First Class Honours degree in Chemical Engineering at the University of Cape Town in 2008 and then went on to read for his PhD at Trinity Hall, University of Cambridge, UK, in the field of Chemical Engineering. He graduated from Cambridge in May 2014 before returning to South Africa to take up a Claude Leon Postdoctoral Research Fellowship at the University of Cape Town, in the Center for Bioprocess Engineering Research. In June 2015 he took up a permanent position as a lecturer at the University of Stellenbosch, in the Department of Process Engineering. 